\title{
Derivation of Breathing Metrics From a Photoplethysmogram at Rest: Machine Learning Methodology
}

Joseph Prinable $^{1}$, BEng; Peter Jones ${ }^{1}$, PhD; David Boland ${ }^{1}$, PhD; Cindy Thamrin ${ }^{2}$, PhD; Alistair McEwan ${ }^{1}, \mathrm{PhD}$

${ }^{1}$ School of Electrical and Information Engineering, The University of Sydney, Darlington, Australia

${ }^{2}$ The Woolcock Institute of Medical Research, The University of Sydney, Glebe, Australia

Corresponding Author:

Joseph Prinable, BEng

School of Electrical and Information Engineering

The University of Sydney

Room 402, Building J03

Maze Crescent

Darlington, 2006

Australia

Phone: 61404035701

Email: joseph.prinable@sydney.edu.au

\begin{abstract}
Background: There has been a recent increased interest in monitoring health using wearable sensor technologies; however, few have focused on breathing. The ability to monitor breathing metrics may have indications both for general health as well as respiratory conditions such as asthma, where long-term monitoring of lung function has shown promising utility.

Objective: In this paper, we explore a long short-term memory (LSTM) architecture and predict measures of interbreath intervals, respiratory rate, and the inspiration-expiration ratio from a photoplethysmogram signal. This serves as a proof-of-concept study of the applicability of a machine learning architecture to the derivation of respiratory metrics.

Methods: A pulse oximeter was mounted to the left index finger of 9 healthy subjects who breathed at controlled respiratory rates. A respiratory band was used to collect a reference signal as a comparison.

Results: Over a 40-second window, the LSTM model predicted a respiratory waveform through which breathing metrics could be derived with a bias value and $95 \%$ CI. Metrics included inspiration time ( -0.16 seconds, -1.64 to 1.31 seconds), expiration time ( 0.09 seconds, -1.35 to 1.53 seconds), respiratory rate ( 0.12 breaths per minute, -2.13 to 2.37 breaths per minute), interbreath intervals ( -0.07 seconds, -1.75 to 1.61 seconds), and the inspiration-expiration ratio $(0.09,-0.66$ to 0.84$)$.

Conclusions: A trained LSTM model shows acceptable accuracy for deriving breathing metrics and could be useful for long-term breathing monitoring in health. Its utility in respiratory disease (eg, asthma) warrants further investigation.
\end{abstract}

(JMIR Mhealth Uhealth 2020;8(7):e13737) doi: $\underline{10.2196 / 13737}$

\section{KEYWORDS}

photoplethysmogram; respiration; asthma monitoring; LSTM

\section{Introduction}

There has been increasing interest in monitoring health using wearable sensors. However, very few technologies have focused on the breathing signal. The ability to monitor breathing may be beneficial for general health and particularly for asthma, which is a health condition that affects over 300 million people globally [1]. Monitoring of lung function using specialized metrics such as peak expiratory flow has been shown to be useful for predicting risk of an asthma episode [2]; however, this can be difficult to perform for patients as it involves forced maneuvers. It remains to be seen whether continuous monitoring of simple breathing metrics such as the interbreath interval (IBI) and the inspiration-expiration (I:E) ratio could provide further information on asthma control [3] and disease status [4].

The availability of a noninvasive sensor that measures breathing continuously and in an ambulatory manner would facilitate studies to establish clinical utility. One sensor of interest is the pulse oximeter that is commonly used in a clinical setting to measure both arterial blood oxygen saturation $\left(\mathrm{SPO}_{2}\right)$ and heart rate. A tidal breathing method exists that also shows promise for clinical prediction [5]; however, these methods are unsuitable 
for continuous monitoring (eg, during walking or exercise). It was recently shown that a pulse oximeter can also be used to continuously monitor respiratory rate in a clinical setting [6]. This is possible because breathing periodicity [6,7] and effort [8] modulate photoplethysmogram (PPG) amplitude, frequency, and baseline wander $[9,10]$. Filtering and feature-based signal processing approaches can be applied to the PPG signal to extract a surrogate respiratory signal. This in turn can be processed to derive breathing rate (BR) with varying degrees of accuracy [7].

Unfortunately, there is poor amplitude correlation between the surrogate respiratory waveform and a gold standard respiratory trace. This poor correlation may make the I:E ratio difficult or impossible to derive using existing methods. In this work, we sought to address this using machine learning. In a previous pilot study [11], we demonstrated how a long short-term memory (LSTM) approach could predict a respiratory waveform from which BR could be derived. LSTM is a type of a recurrent neural network that can capture long-term, time-based dependencies in data [12]. Through the LSTM, we showed that the Pearson correlation coefficient between the derived respiratory waveform and a pneumotachograph trace had similarly high $r$ values $(r>0.8)$ to existing methods. In this paper, we built on this study by investigating the accuracy to which IBI, I:E ratio, and BR respiratory metrics can be attained from a PPG-derived surrogate respiratory waveform using an LSTM. We show that, in comparison to existing approaches, we can derive breathing metrics to a higher degree of accuracy from a pulse oximeter.

\section{Methods}

\section{Datasets}

\section{Data Collection}

Measurements were recorded from a group of 10 healthy participants who provided informed consent. The protocol for this study was approved by Northern Sydney Local Health District Human Research Ethics Committee (LNR/16/HAWKE/99 ethics approval). Participants conducted 5 randomized breathing serials at a rate of $6,8,10,12$, or 14 breaths per minute (BPM). Each serial was conducted for 5 minutes. Each participant was coached to breath one full inhalation and exhalation in time with a visual prompt.

An Alice PDx (Philips Respironics, Murrysville, PA) portable sleep diagnostic system was used to measure physiological signals during this study. The supplied pulse oximeter was attached to the index finger of the nonmaster hand, allowing the capture of a raw PPG trace, $\mathrm{SPO}_{2}$, and pulse rate data. The Alice PDx reported calculated values for $\mathrm{SPO}_{2}$ and pulse rate 3 times per second. PPG signals were sampled at $75 \mathrm{~Hz}$.
Respiratory inductance plethysmography is a method to measure relative tidal volume (RTV) as a function of the chest and abdominal wall movement [13]. In this study, inductance bands were placed around the abdomen and ribcage according to the manufacturer's guidelines, allowing RTV to be estimated as the weighted sum of the chest and abdominal wall inductance signals. The Alice PDx system reported an RTV signal based on the contribution of both respiratory bands and was captured at $100 \mathrm{~Hz}$.

\section{Description of Available Features}

The Alice PDx system outputs three independent time series: PPG, $\mathrm{SPO}_{2}$, and pulse rate. The $\mathrm{SPO}_{2}$, processed PPG, and pulse rate signals were up-sampled to $25 \mathrm{~Hz}$ while the RTV was down-sampled to $25 \mathrm{~Hz}$ before normalizing between \pm 1 . The sampling rate of $25 \mathrm{~Hz}$ was selected to ensure respiratory rate accuracy $[7,14]$ and so that all time series data had the same time scale.

In addition to the three time series given by the Alice PDx system, a bandpassed PPG time series was generated by passing the original PPG signal through a sixth order Butterworth bandpass filter with a center frequency corresponding to the respiratory rate of the signal with a bandwidth of $0.002 \mathrm{~Hz}$. This additional time series was included because our previous findings suggested that this feature could improve model prediction [11].

Altogether, the available features used within our model are as follows:

- $\quad$ Feature 1: PPG

- Feature 2: bandpassed PPG

- Feature 3: $\mathrm{SPO}_{2}$

- Feature 4: pulse rate

We previously determined experimentally that the inclusion of $\mathrm{SPO}_{2}$ and pulse rate values helped inform the network when decoupling between the pulse signal and respiratory signal occurs [11]. The exact underlying physiological mechanisms are unclear.

\section{Derivation of a Respiratory Waveform Time Series}

For comparison purposes, RRest toolbox [15] was used to extract respiratory waveforms from a PPG using 10 feature-based and filter-based algorithms as shown in Figure 1. The resulting respiratory waveforms were temporally aligned to correspond with the reference respiratory waveform in the test set for comparison purposes. The techniques used to derive the respiratory waveforms, as well as our LSTM method, are described in Table 1. 
Figure 1. Using existing filter-based and feature-based methods, 10 relative respiratory waveforms were derived from a photoplethysmogram (PPG) signal, and another relative respiratory waveform was derived using a long short-term memory (LSTM) that accepts PPG, arterial blood oxygen saturation (SPO2), band-passed (BP) PPG, and pulse rate inputs. BR: breathing rate; I:E: inspiration-expiration ratio; IBI: interbreath interval.

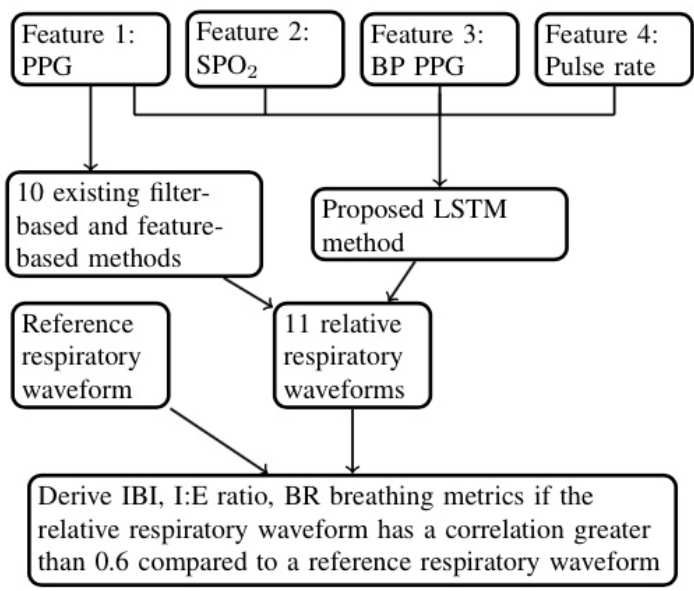

Table 1. Techniques for the extraction of respiratory signals from a photoplethysmogram (adapted from Charlton et al [15]).

\begin{tabular}{|c|c|}
\hline Respiratory signal & Description \\
\hline \multicolumn{2}{|l|}{ Filter-based } \\
\hline $\mathrm{X}_{\mathrm{A} 1}$ & Bandpass filter between plausible respiratory frequencies \\
\hline $\mathrm{X}_{\mathrm{A} 2}$ & $\begin{array}{l}\text { Maximum amplitude of the } \mathrm{CWT}^{\mathrm{a}} \text { within plausible cardiac frequencies } \\
\text { (30-220 beats per minute) [16] }\end{array}$ \\
\hline $\mathrm{X}_{\mathrm{A} 3}$ & $\begin{array}{l}\text { The frequency corresponding to the maximum amplitude of the CWT } \\
\text { within plausible cardiac frequencies [16] }\end{array}$ \\
\hline \multicolumn{2}{|l|}{ Feature-based } \\
\hline $\mathrm{X}_{\mathrm{B} 1}$ & Mean amplitude of troughs and proceeding peaks [7] \\
\hline $\mathrm{X}_{\mathrm{B} 2}$ & Difference between the amplitudes of troughs and proceeding peaks [17] \\
\hline $\mathrm{X}_{\mathrm{B} 3}$ & Time interval between consecutive troughs [17] \\
\hline $\mathrm{X}_{\mathrm{B} 4}$ & Mean signal value between consecutive troughs [18] \\
\hline $\mathrm{X}_{\mathrm{B} 5}$ & Peak amplitude [17] \\
\hline $\mathrm{X}_{\mathrm{B} 6}$ & Trough amplitude [18] \\
\hline $\mathrm{X}_{\mathrm{B} 10}$ & $\begin{array}{l}\mathrm{PPG}^{\mathrm{b}} \text { pulse width estimation using a wave boundary detection algorithm } \\
\text { [19] }\end{array}$ \\
\hline \multicolumn{2}{|c|}{ Machine learning-based } \\
\hline $\mathrm{X}_{\mathrm{LSTM}}{ }^{\mathrm{c}}$ & Proposed LSTM method \\
\hline
\end{tabular}

${ }^{\mathrm{a}} \mathrm{CWT}$ : continuous wavelet transform.

${ }^{\mathrm{b}} \mathrm{PPG}$ : photoplethysmogram.

${ }^{\mathrm{c}}$ LSTM: long short-term memory.

\section{LSTM Architecture and Parameters}

We propose the use of an LSTM model as an alternative to the signal processing methods described in Table 1 . In this section, we discuss our training and validation procedures to determine an appropriate LSTM architecture to predict a respiratory waveform.

The core component of an LSTM architecture is a memory cell whose characteristics allow long-term data dependencies to be captured. A single LSTM cell uses gate mechanisms to forget irrelevant parts of a previous state, selectively update the current cell state, and to output the cell state [12]. Each cell contains a number of hidden units that define the dimensionality of both the current and output states. Increasing hidden units within a model may lead to overfitting. Conversely, reducing hidden units below a certain threshold will not allow a model to be trained. 


\section{Hyperparameter Search}

We first conducted a structured, though nonexhaustive, hyperparameter search to determine suitable values for our final LSTM architecture. We then performed more extensive training to maximize the performance of our final architecture.

\section{Hyperparameter Exploration}

An open-source Python 3.5 library called TensorFlow r1.3 was used to train the LSTM model on a Dell Optiplex D810 (i7, 32 GB RAM; Dell Inc, Round Rock, TX) and two Titan Xp (Nvidia Corp, Santa Clara, CA) graphics processing units (GPUs).

The AdamOptimizer class of Tensorflow was used to train the LSTM using a learning rate of 0.0005 for 100 epochs with a batch size of 128 . We explored the effect of changing the amount of cells $(100,300,500)$, hidden units within a cell (500, $1500,2500)$, and layers $(1,2,3)$ and compared the results against a default model containing 100 cells, 500 hidden units, and a single layer. For this study, cells were layered sequentially two times to improve model accuracy and robustness [20]. The dropout layer was placed between each layer with a dropout rate of 0.5 to reduce overfitting [21]. There was a single dense, fully connected layer at the end.

To minimize training time for hyperparameter exploration, 4 smaller training datasets were created from the original 45 unique datasets ( 9 participants, each with 5 breathing serials). These datasets contained data from 1 participant (7), 3 participants $(3,5,7), 5$ participants $(1,3,5,7,9)$, or $9(1,2,3$, $4,5,6,7,8,9)$ participants. This allowed us to compare model performance as the number of participants increased for the various configurations. To further reduce training time, each dataset was reduced to 1 minute of data, splitting $70 \%, 15 \%$, and $15 \%$ into training, validation, and test sets, respectively. To assess the performance of the model, we conducted 5-fold cross validation. To reduce computational time that typically results in higher error bias but lower variability, we chose 5 folds over 10 folds [22]. We investigated permutations of the available features and found that accuracy increased with the number of features with a minimal cost in terms of execution time.

Table 2 shows the training time in minutes as a function of participants and the hyperparameter. The Pearson correlation coefficients between the derived and reference respiratory waveforms are plotted as a function of increasing number of participants for the chosen cell values (Table 2) in Figure 2A, hidden unit values in Figure 2B, and layer values in Figure 2C. The highest correlation was achieved with 300 cells and 2 layers for 9 participants. For hidden units, the correlation was similar between the quantities, with 2500 hidden units only slightly better than 500 ( 0.786 vs 0.788 ). Due to the minimal difference, the latter was selected as it required significantly less training time (211 minutes vs 1213 minutes) for comparable performance.

Table 2. Training time (minutes) for the hyperparameter search.

\begin{tabular}{cllll}
\hline Hyperparameters & 1 participant & 3 participants & 5 participants & 9 participants \\
\hline Cells & 24 & 75 & 110 & 208 \\
100 & 54 & 200 & 272 & 505 \\
300 & 84 & 313 & 542 & 932 \\
500 & & & & \\
Hidden units & 24 & 69 & 150 & 211 \\
500 & 52 & 161 & 264 & 482 \\
1500 & 131 & 402 & 665 & 1213 \\
2500 & & & & 220 \\
Layers & 24 & 65 & 116 & 366 \\
1 & 35 & 125 & 190 & 470 \\
3 & 48 & 158 & 271 & \\
\hline
\end{tabular}


Figure 2. Pearson correlation values between derived and reference respiratory waveforms, given a dataset containing $\mathrm{n}$ participants, for a long short-term memory (LSTM) with (A) cells of size 100, 300, and 500; (B) hidden units of size 500, 1500, and 2500; (C) layers of size 1, 2 , and 3.

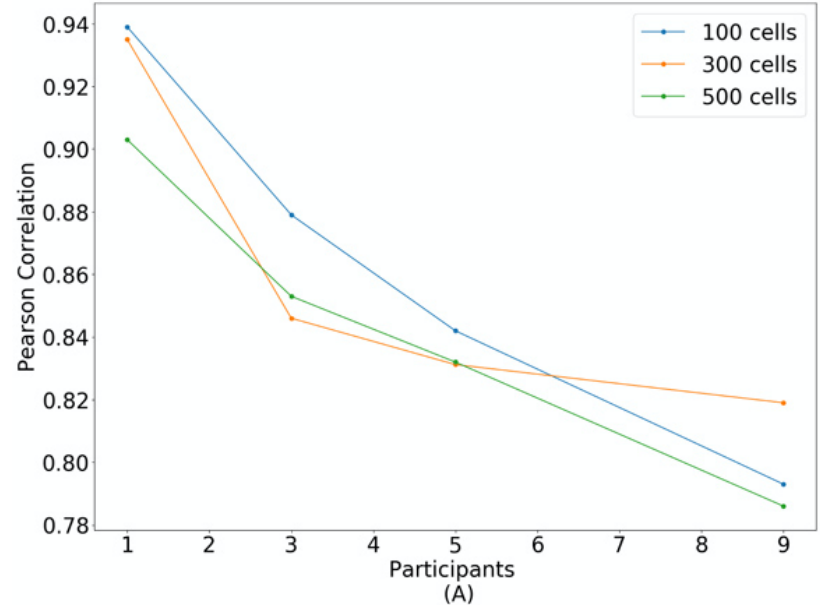

(A)

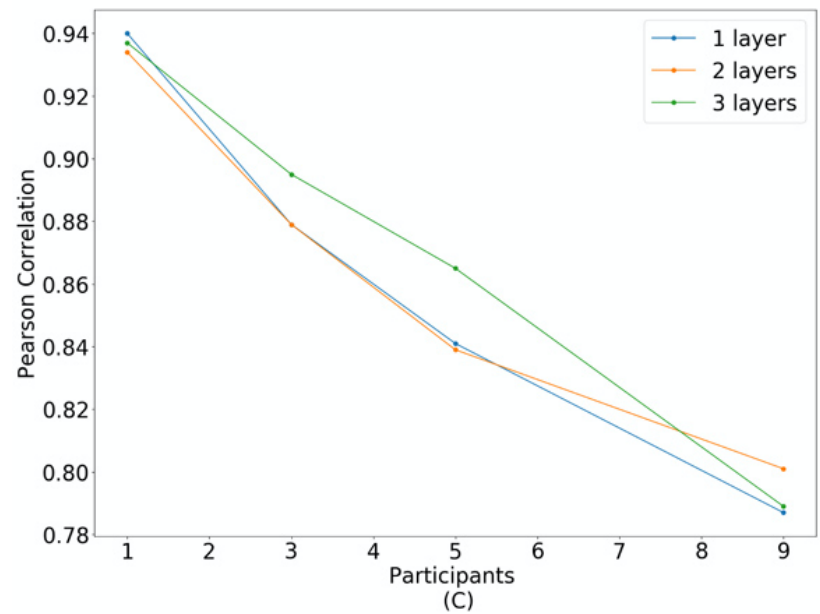

\section{Final Model Training}

The final model had around 8257 trainable parameters consisting of 300 cells, 2 layers, and 16 hidden layers. To train our final model, we used AdamOptimizer with an initial learning rate of 0.02 and batch size of 256 . We conducted 5-fold cross validation with approximately 223,786 training examples per fold with early stopping.

\section{Extraction of Breathing Metrics}

We defined a valid window when the Pearson correlation coefficient was $>0.6$ between the gold standard respiratory waveform and derived tidal volume waveform (TVW) in the window. For test sets that contained valid windows, we extracted peaks and troughs in MATLAB R2016b (MathWorks Inc, Natick, MA). To find the maximum points, 'findpeaks' was used, and we used a linear search algorithm to find the global minimum between 2 consecutive peaks. Using the peak and trough data, we extracted the following: IBI (the period in seconds between 2 consecutive peaks within the TVW signal), inspiration time (period in seconds between a trough and peak within the TVW signal), expiration period (period in seconds between a peak and trough within the TVW signal), and I:E (ratio between consecutive inspiration time and expiration period).

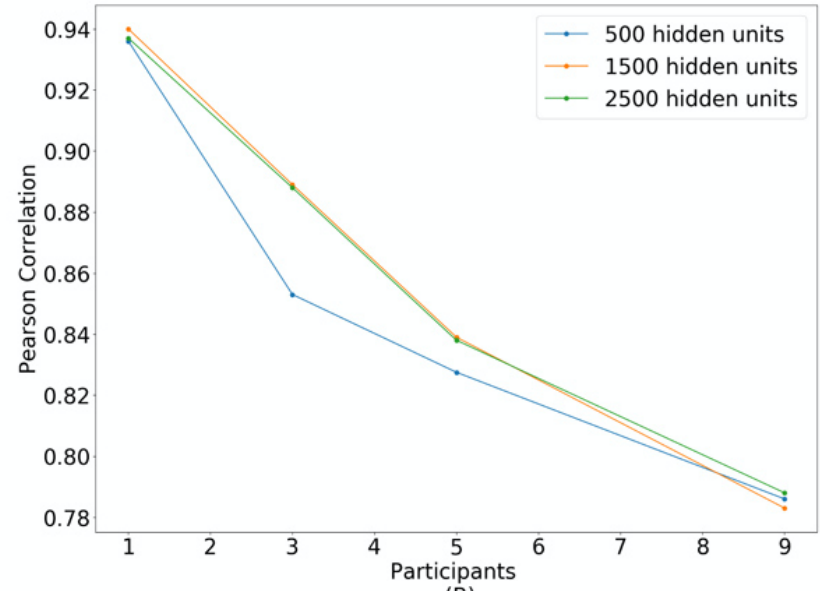

(B)

We then evaluated the Bland-Altman agreement [13] between the derived respiratory metrics to reference metrics.

Additionally, the root mean square error between hypothesized RTV signal $y(t)$ and the true RTV Y (t) was calculated for each person and respiratory rate and subsequently averaged across the 5 folds.

\section{Results}

\section{Data Collection}

Data were acquired from 10 healthy subjects. One subject was excluded because of incomplete recordings due to an SD card save error on the Alice PDx. Therefore, data for 9 subjects were analyzed. The median (lower, upper quartiles) age of the analyzed subjects was 28 years (24.5 to 33.0 years). Median BMI was $23.59 \mathrm{~kg} / \mathrm{m}^{2}$ (21.28 to $\left.30.04 \mathrm{~kg} / \mathrm{m}^{2}\right)$, and 3 subjects $(3 / 9,33 \%)$ were female. In total, we recorded 3.75 hours of data, consisting of 5 minutes $* 5$ breathing rates $* 9$ participants.

\section{Model Validation}

The weights and biases were saved for each epoch during training. Training was stopped when the validation error diverged to avoid overfitting. Early stopping occurred when the validation cost did not improve for 5 epochs. 


\section{Derivation of Breathing Metrics}

In total, 225 unique test sets were created from 9 participants, at 5 respiratory rates, over 5 folds. Each test set was a window of 1000 samples ( 40 seconds) in length. We plotted the number of valid windows as a function of increasing Pearson correlation coefficients between derived and reference respiratory waveforms in 0.2 increments in Figure 3. For a Pearson correlation coefficient $\geq 0.6$, our approach, $X_{\mathrm{LSTM}}$, was valid for $191 / 225$ (85\%) windows, while the next highest performing algorithm, $\mathrm{X}_{\mathrm{A} 1}$, was valid for 128/225 (57\%) windows, followed by $X_{A 2}$, which was valid for $119 / 225$ (53\%) windows. Other algorithms were excluded from further analysis due to a small percentage of valid windows: $21 / 225(9 \%)$ for $\mathrm{X}_{\mathrm{A} 3}, 56 / 225$ (25\%) for $\mathrm{X}_{\mathrm{B} 1}, 38 / 225(17 \%)$ for $\mathrm{X}_{\mathrm{B} 2}, 36 / 225(16 \%)$ for $\mathrm{X}_{\mathrm{B} 3}$, $23 / 225(10 \%)$ for $X_{B 4}, 65 / 225(29 \%)$ for $X_{B 5}, 52 / 225(23 \%)$ for $\mathrm{X}_{\mathrm{B} 6}$, and $11 / 225(5 \%)$ for $\mathrm{X}_{\mathrm{B} 10}$.
Breathing metrics were averaged over each 40-second test set. The mean (SD) between derived and gold standard metrics and their associated $t$ test results are shown in Table 3 . The Bland-Altman agreement between derived and gold standard metrics for all subjects and respiratory rates are reported in Table 4. In the case of $\mathrm{X}_{\mathrm{LSTM}}$, a Savitzky-Golay filter was used to smooth the derived respiratory waveform prior to extracting the breathing metrics.

The Bland-Altman plot for the derived breathing metrics of inspiration time, expiration period, IBI, BR, and I:E across all participants (1-9) and all respiratory rates $(6,8,10,12,14)$ using $\mathrm{X}_{\mathrm{LSTM}}$ is shown in Figure 4. For comparison purposes, we report the Bland-Altman plot for derived respiratory rate across all participants and all respiratory rates using the highest performing algorithm found by Charlton et al [7] in Figure 5.

Figure 3. Number of valid windows as a function of increasing Pearson correlation coefficients between derived and reference respiratory waveforms in 0.2 increments. For an explanation of the variables please refer to Table 1.

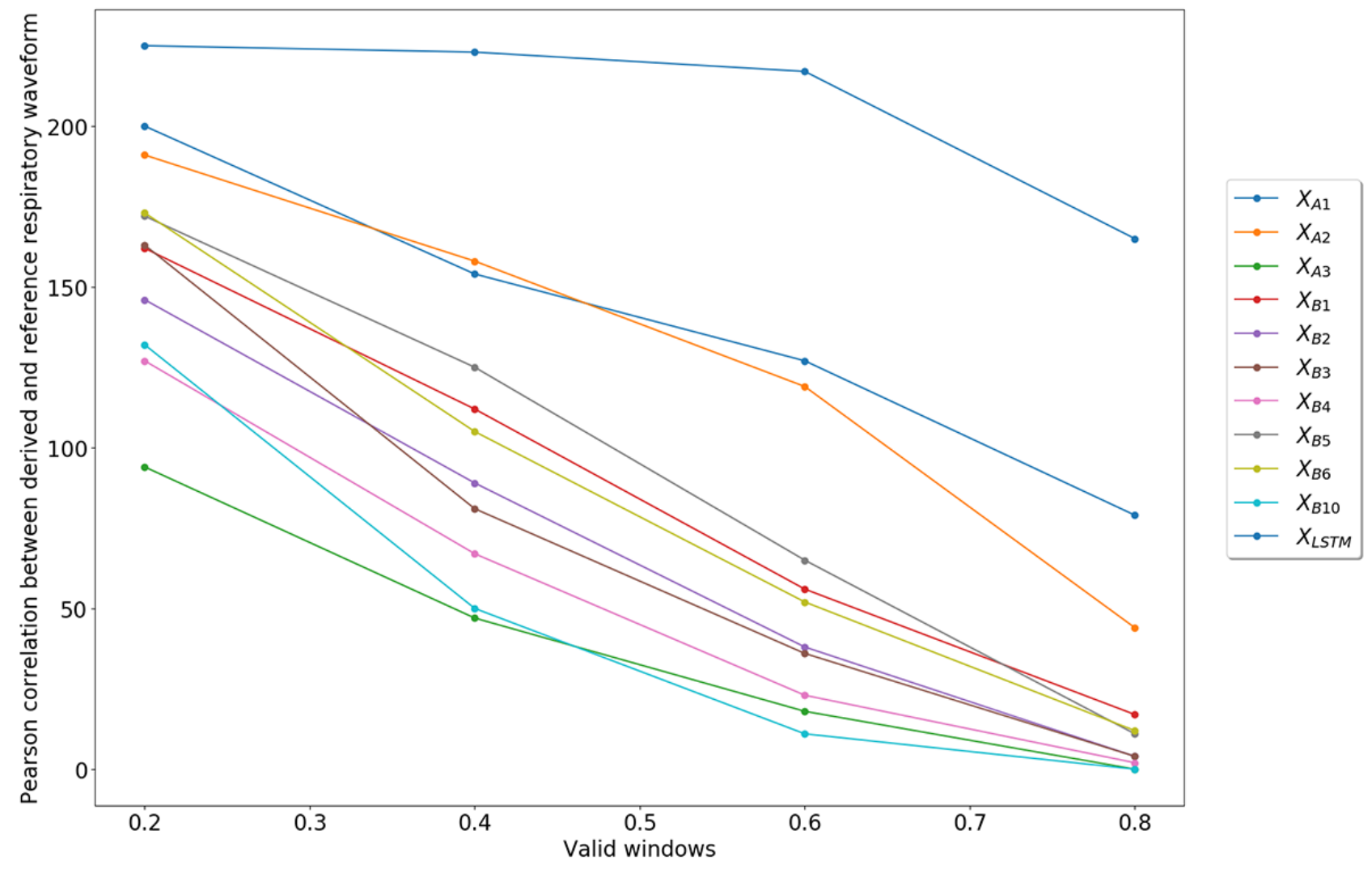


Table 3. Breathing metrics for the reference respiratory band, $X_{\mathrm{LSTM}}, \mathrm{X}_{\mathrm{A} 1}$, and $\mathrm{X}_{\mathrm{A} 2}$ methods, with their associated paired $t$ test results.

\begin{tabular}{|c|c|c|c|c|c|c|c|c|c|c|}
\hline \multirow{2}{*}{$\begin{array}{l}\text { Breathing } \\
\text { metrics }\end{array}$} & \multirow{2}{*}{$\begin{array}{l}\text { Respiratory band, } \\
\text { mean (SD) }\end{array}$} & \multicolumn{3}{|c|}{$\mathrm{X}_{\mathrm{LSTM}^{\mathrm{a}}}{ }^{\mathrm{a}}$} & \multicolumn{3}{|l|}{$\mathrm{X}_{\mathrm{A} 1}$} & \multicolumn{3}{|l|}{$\mathrm{X}_{\mathrm{A} 2}$} \\
\hline & & $\begin{array}{l}\text { Mean } \\
(\mathrm{SD})\end{array}$ & $\mathrm{t}_{180}$ test & $P$ & Mean (SD) & $\mathrm{t}_{126}$ test & $P$ & Mean (SD) & $\mathrm{t}_{118}$ test & $P$ \\
\hline $\begin{array}{l}\text { Tinsp }{ }^{b} \text { (sec- } \\
\text { onds) }\end{array}$ & $3.28(1.29)$ & $\begin{array}{l}3.14 \\
(1.15)\end{array}$ & 2.92 & 0.004 & $3.46(1.31)$ & 1.65 & 0.103 & $3.40(1.42)$ & 0.02 & 0.99 \\
\hline $\begin{array}{l}\text { Texp }{ }^{c}(\mathrm{sec}- \\
\text { onds) }\end{array}$ & $3.13(1.01)$ & $\begin{array}{l}3.19 \\
(1.05)\end{array}$ & -1.68 & 0.095 & 3.38 (1.09) & -3.24 & 0.002 & $3.10(0.95)$ & -1.44 & 0.152 \\
\hline $\mathrm{BR}^{\mathrm{d}}\left(\mathrm{BPM}^{\mathrm{e}}\right)$ & $10.28(2.72)$ & $\begin{array}{l}10.41 \\
(2.74)\end{array}$ & -1.39 & 0.167 & $9.69(2.73)$ & 1.93 & 0.056 & $10.35(2.95)$ & -0.46 & 0.649 \\
\hline $\mathrm{IBI}^{\mathrm{f}}$ (seconds) & $6.40(1.98)$ & $\begin{array}{l}6.33 \\
(1.96)\end{array}$ & 1.12 & 0.262 & $6.84(2.09)$ & -2.18 & 0.031 & $6.50(2.09)$ & -1.73 & 0.086 \\
\hline $\mathrm{I}: \mathrm{E}^{\mathrm{g}}$ & $1.01(0.36)$ & $\begin{array}{l}1.09 \\
(0.43)\end{array}$ & -3.09 & 0.002 & $1.03(0.40)$ & -2.68 & 0.008 & $1.00(0.29)$ & -1.50 & 0.135 \\
\hline
\end{tabular}

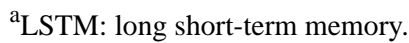

$\mathrm{b}_{\text {Tinsp: inspiration time. }}$

${ }^{\mathrm{c}}$ Texp: expiration period.

${ }^{\mathrm{d}} \mathrm{BR}$ : breathing rate.

${ }^{\text {BPM: breaths per minute. }}$

${ }^{\mathrm{f}} \mathrm{IBI}$ : interbreath interval.

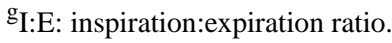


Table 4. Derived breathing metrics using the $\mathrm{X}_{\mathrm{LSTM}}, \mathrm{X}_{\mathrm{A} 1}$, and $\mathrm{X}_{\mathrm{A} 2}$ methods and associated statistical analyses.

\begin{tabular}{|c|c|c|c|c|c|c|}
\hline \multirow[t]{2}{*}{ Method } & \multirow[t]{2}{*}{ Bland-Altman $r^{2}$} & \multirow[t]{2}{*}{$P$} & \multicolumn{2}{|c|}{ Absolute } & \multicolumn{2}{|l|}{ Relative } \\
\hline & & & Bias & $95 \% \operatorname{LoA}^{\mathrm{a}}$ & Bias (\%) & $95 \%$ LoA \\
\hline \multicolumn{7}{|c|}{ Tinsp (seconds) ${ }^{b}$} \\
\hline $\mathrm{X}_{\mathrm{LSTM}}{ }^{\mathrm{c}}$ & 0.70 & $<.001$ & -0.16 & -1.64 to 1.31 & -3.70 & -38.44 to 31.05 \\
\hline $\mathrm{X}_{\mathrm{A} 1}$ & 0.74 & $<.001$ & -0.11 & -1.51 to 1.30 & -2.35 & -35.65 to 30.95 \\
\hline $\mathrm{X}_{\mathrm{A} 2}$ & 0.74 & $<.001$ & -0.01 & -1.46 to 1.46 & -0.22 & -33.34 to 32.90 \\
\hline \multicolumn{7}{|c|}{ Texp (seconds) ${ }^{d}$} \\
\hline $\mathrm{X}_{\mathrm{LSTM}}$ & 0.54 & $<.001$ & 0.09 & -1.35 to 1.53 & 2.35 & -31.84 to 36.55 \\
\hline $\mathrm{X}_{\mathrm{A} 1}$ & 0.41 & $<.001$ & 0.25 & -1.45 to 1.95 & 6.41 & -32.82 to 45.63 \\
\hline $\mathrm{X}_{\mathrm{A} 2}$ & 0.43 & $<.001$ & 0.10 & -1.39 to 1.59 & 2.70 & -36.34 to 41.73 \\
\hline \multicolumn{7}{|l|}{$\mathbf{B R}^{\mathrm{e}}\left(\mathbf{B P M}^{\mathbf{f}}\right)$} \\
\hline $\mathrm{X}_{\mathrm{LSTM}}$ & 0.83 & $<.001$ & 0.12 & -2.13 to 2.37 & 1.22 & -23.63 to 26.07 \\
\hline $\mathrm{X}_{\mathrm{A} 1}$ & 0.92 & $<.001$ & -0.13 & -1.68 to 1.41 & -1.38 & -18.42 to 15.65 \\
\hline $\mathrm{X}_{\mathrm{A} 2}$ & 0.88 & $<.001$ & 0.04 & -1.94 to 2.02 & 0.14 & -19.35 to 19.62 \\
\hline \multicolumn{7}{|c|}{ IBI $^{\mathrm{g}}$ (seconds) } \\
\hline $\mathrm{X}_{\mathrm{LSTM}}$ & 0.82 & $<.001$ & -0.07 & -1.75 to 1.61 & -0.98 & -22.62 to 20.66 \\
\hline $\mathrm{X}_{\mathrm{A} 1}$ & 0.88 & $<.001$ & 0.14 & -1.31 to 1.60 & 2.08 & -16.55 to 20.70 \\
\hline $\mathrm{X}_{\mathrm{A} 2}$ & 0.91 & $<.001$ & 0.10 & -1.13 to 1.33 & 1.37 & -16.20 to 18.94 \\
\hline \multicolumn{7}{|l|}{$\mathbf{I}: \mathbf{E}^{\mathbf{h}}$} \\
\hline $\mathrm{X}_{\mathrm{LSTM}}$ & 0.30 & $<.001$ & 0.09 & -0.66 to 0.84 & 9.91 & -63.89 to 83.70 \\
\hline $\mathrm{X}_{\mathrm{A} 1}$ & 0.11 & $<.001$ & 0.09 & -0.68 to 0.87 & 6.65 & -61.43 to 74.73 \\
\hline $\mathrm{X}_{\mathrm{A} 2}$ & 0.04 & $<.001$ & 0.05 & -0.62 to 0.71 & 3.41 & 63.89 to 70.72 \\
\hline
\end{tabular}

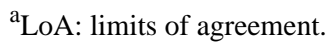

${ }^{\mathrm{b}}$ Tinsp: inspiration time.

${ }^{\mathrm{c}}$ LSTM: long short-term memory.

${ }^{\mathrm{d}}$ Texp: expiration period.

${ }^{\mathrm{e}} \mathrm{BR}$ : breathing rate.

${ }_{\text {f }}$ PM: breaths per minute.

${ }^{\mathrm{g}}$ IBI: interbreath interval.

$\mathrm{h}_{\text {I:E: inspiration:expiration ratio. }}$ 
Figure 4. Bland-Altman plots for (A) inspiration time (seconds), (B) expiration time (seconds), (C) interbreath interval (seconds), (D) breathing rate (breaths per minute), and (E) inspiration:expiration ratio using the LSTM method.

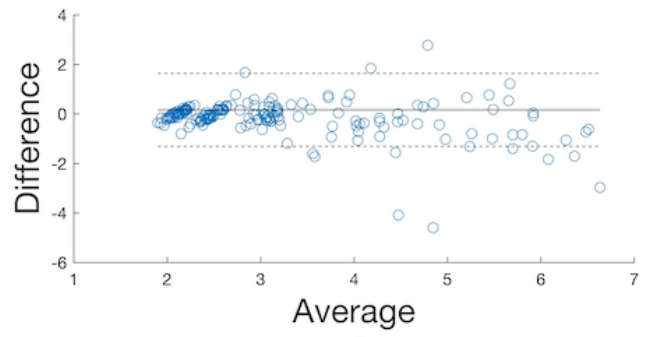

(A)

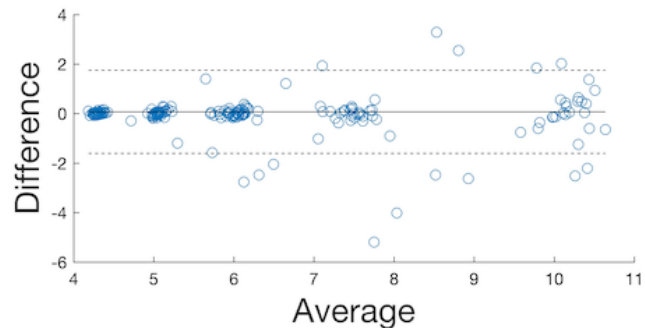

(C)

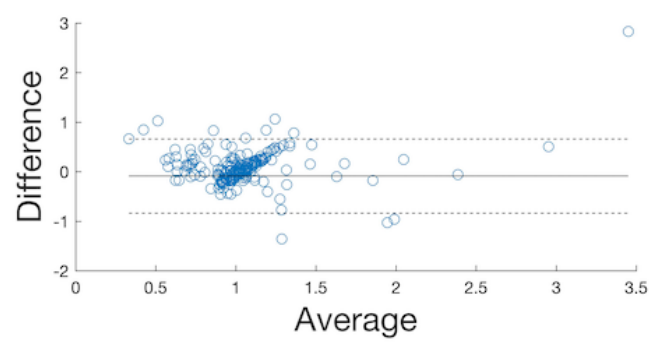

(E)

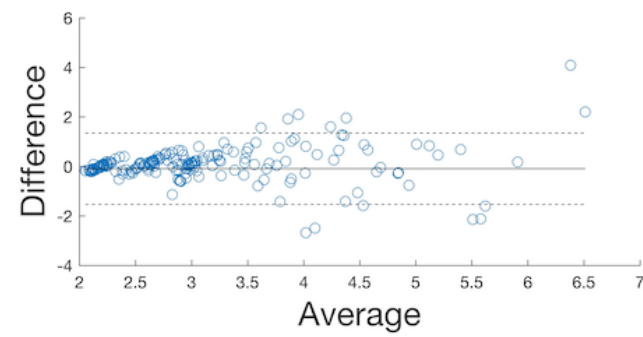

(B)

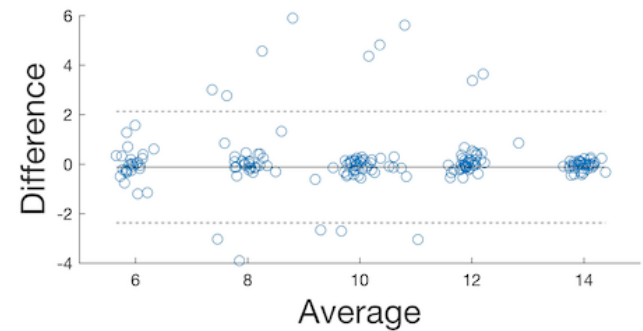

(D) 
Figure 5. Bland-Altman plot for the highest performing algorithm $\left(\mathrm{X}_{\mathrm{B} 1,2,3} \mathrm{E}_{\mathrm{T} 4} \mathrm{~F}_{\mathrm{M} 1}\right)$ found by Charlton et al [7].

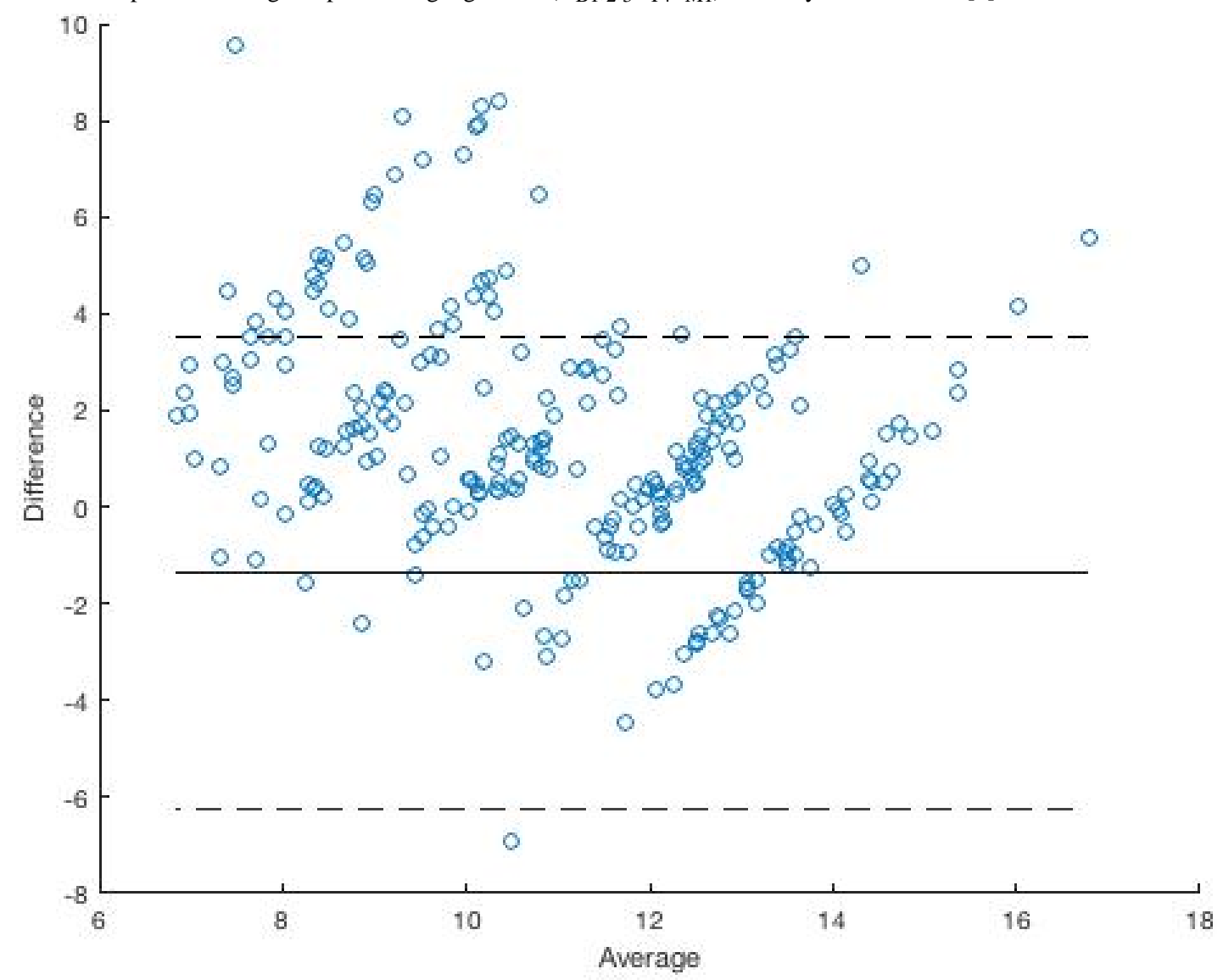

Our model consistently performed comparably to the other methods, showing similar agreement (lower bias) and variability (narrower limits of agreement). The relative bias for our model was $<4 \%$ for all breathing metrics examined except for I:E ratio (at $9.9 \%$ ), which is within the limits of accuracy of existing standards on the estimation of breathing metrics using conventional methods [5], although the limits of variability are wide.

The differences for inspiration time are bound within the $95 \%$ CIs for average inspiration periods $<4$ seconds. Distinct clustering can be seen around an inspiration period of 2 seconds (Figure 4A). The differences for expiration period are bound within the $95 \%$ CIs for average expiration periods of 2-3 seconds (Figure 4B). For IBI, 4 distinct clusters occur corresponding to intervals of $4,5,6$, and 7 seconds; however, the clustering weakens above 9 seconds (Figure 4C). For BR, 5 distinct clusters are formed corresponding to expected BRs of $6,8,10$, 12, and 14 BPM (Figure 4D). There is noticeable clustering for I:Es of 0.8-1 (Figure 4E).

To quantify the accuracy of our model and provide a metric for future comparisons, we report the root mean square error over all participants and respiratory rates for $\mathrm{X}_{\mathrm{LSTM}}$ for inspiration time (0.77 seconds), expiration period (0.74 seconds), IBI (0.8377 seconds), BR (0.86 BPM), and I:E (1.15).

\section{Discussion}

\section{Principal Findings}

In this work, we were interested in determining the feasibility of finding continuous measures of inspiration time, expiration period, IBI, BR, and I:E metrics from a PPG. We showed how an LSTM architecture could be used to predict these metrics for 191/225 (85\%) test sets comprised of 9 participants at a respiratory rate of $6,8,10,12$, or $14 \mathrm{BPM}$. We conducted Bland-Altman analyses and found the LSTM was able to predict the average inspiration time of -0.16 seconds $(-1.64$ to 1.31 seconds) and expiration period of 0.09 seconds ( -1.35 to 1.53 seconds) over a 40-second window. The LSTM was able to predict an I:E ratio of 0.09 ( -0.66 to 0.84$)$, although this was poorly correlated with reference values. However, this is the first time this metric is being reported in the literature as measured from a pulse signal.

The LSTM model was trained to minimize the error between derived and reference respiratory waveforms and was then able to generalize the breathing characteristics of 9 subjects and predict future respiratory waveforms based on PPG data. The ability to "see and learn" a reference signal presents a distinct advantage over existing methods. Through this approach, it was possible to determine the continuous average breathing metrics of inspiration time, expiration period, IBI, and BR for the majority of time (85\%), exceeding a Pearson correlation threshold of 0.6 . In contrast, these breathing metrics could only be derived, at best, around half the time $(56 \%$ in the case of 
$\mathrm{X}_{\mathrm{A} 1}$ ) using existing feature-based and filter-based algorithms that did not rely on any previous reference data. While we directly compared the performance of $\mathrm{X}_{\mathrm{A} 1}$ and $\mathrm{X}_{\mathrm{A} 2}$ to the LSTM method, other methods were excluded from this analysis due to the fact that the correlation between the derived respiratory waveform and the gold standard was $<0.6$ more than $80 \%$ of the time. Feature-based techniques $\left(\mathrm{X}_{\mathrm{B} 1}-\mathrm{X}_{\mathrm{B} 6}, \mathrm{X}_{\mathrm{B} 10}\right)$ have performed well in previous respiratory rate algorithm assessments by Charlton et al [7] and would likely have similar performance on this dataset. In the cases where breathing metrics could be extracted for $\mathrm{X}_{\mathrm{A} 1}$ and $\mathrm{X}_{\mathrm{A} 2}$, we found that the metrics of inspiration time, expiration period, and I:E were poorly correlated with the reference metrics, as shown in Table 4.

We conducted Bland-Altman analysis on the highest performing algorithm $\mathrm{X}_{\mathrm{B} 1,2,3} \mathrm{E}_{\mathrm{T} 4} \mathrm{~F}_{\mathrm{M} 1}$ found by Charlton et al [7] in his comparison of classical signal processing algorithms for PPG. The bias in our dataset compared to those in the dataset used by Charlton et al [7] was higher (-1.12 vs 1). However, the $95 \%$ limits of agreement (BPM) was lower ( -2.4 to 2.1 vs -5.1 to 7.2). $X_{\mathrm{LSTM}}$ compares favorably to $\mathrm{X}_{\mathrm{B} 1,2,3} \mathrm{E}_{\mathrm{T} 4} \mathrm{~F}_{\mathrm{M} 1}$ with similar bias $(0.12$ vs -1.10$)$ and a smaller $95 \%$ limits of agreement (BPM; -2.13 to 2.37 vs -2.63 to 2.44 ). The bias in our model compares well against existing standards on breathing metric estimation using conventional methods, which stipulate an accuracy of at least $2 \%$ for respiratory rate. It is worth noting that the standards are formulated for infant populations who breath faster. The wide variability seen in our model could be improved, although it is lower than that obtained from other methods examined. The high degree of variability could arise from differences in accuracy with different respiratory rates. While there is insufficient data from this study to ascertain this, it justifies use of longer-term data collection for further investigation.

The hyperparameters for the LSTM model were chosen in a structured, although non-exhaustive, manner by comparing a change in the number of cells, hidden units, or layers to a fixed model. Figures 2-4 show a decreasing trend in the correlation between the LSTM-derived respiratory waveform and the reference waveform as the number of participants increased. This trend occurred irrespective of the number of cells, hidden units, or layers. This may be accounted for, in part, by the complexity for which the LSTM model must account as the participant population increases. In the specific case of 300 cells, the correlation curve decreased quasi-exponentially. However, in the case of hidden units and layers, the correlation curve decreased quasi-linearly. It remains to be seen if the minimum correlation is bound between derived and reference respiratory waveforms for a given population. The findings of this paper show that our previous network parameter was much larger than required [11].

In this work, we used the following 4 features: PPG, filtered $\mathrm{PPG}, \mathrm{SPO}_{2}$, and pulse rate. We did not conduct feature selection, which may have helped to improve the overall model performance. It would be useful to see the effect of removing the filtered PPG signal feature to reduce additional preprocessing time and computational power.

Due to a limited participant population, we did not conduct leave-one-out participant cross validation. The shape of each respiratory waveform varied from person to person, and it is unlikely that the LSTM model derived in this work would be able to predict respiratory metrics from an unseen participant. However, with a larger training population, the LSTM model may be exposed to enough data to enable the accurate prediction of respiratory metrics in an unseen participant. Previously, we found that participants would prefer that a wearable sensor device have a watch form factor [23]. In this paper, we did not look at the feasibility of implementing an LSTM in this type of form factor. Currently, LSTM training requires GPU-grade computational power. With current low-power Bluetooth low energy devices $[11,24,25]$, it may be possible to acquire PPG data and stream real-time data to a cloud-based GPU server to run online training. Once the weights and biases of the LSTM architecture are found, it may also be possible for an embedded platform to perform the required processing to obtain real-time breathing metric predictions. At present, field-programmable gate arrays can be used for real-time predictions and benefit from low latency and low power consumption [26]. Additionally, the field-programmable gate array architecture is reconfigurable. This would allow any potential device to be individually tailored to a specific model.

\section{Conclusion}

This paper presents the feasibility of monitoring simple breathing metrics such as the IBI, BR, inspiration time, expiration period, and I:E for a person at rest. We hope this proof-of-concept paper will inspire future research to collect further data and develop more powerful machine learning algorithms. In the future, it may also be possible to derive these metrics from a wristworn device that contains a pulse oximeter and accelerometer for a person at rest and support potential longitudinal studies to determine if these metrics can provide further information on asthma type [3] and provide any clinical utility [4].

\section{Conflicts of Interest}

None declared.

\section{References}

1. World Health Organization. World health statistics 2010. URL: https://www.who.int/whosis/whostat/2010/en/ [accessed 2020-05-15]

2. Frey U, Brodbeck T, Majumdar A, Taylor DR, Town GI, Silverman M, et al. Risk of severe asthma episodes predicted from fluctuation analysis of airway function. Nature 2005 Dec 01;438(7068):667-670. [doi: 10.1038/nature04176] [Medline: 16319891] 
3. Raoufy MR, Ghafari T, Darooei R, Nazari M, Mahdaviani SA, Eslaminejad AR, et al. Classification of Asthma Based on Nonlinear Analysis of Breathing Pattern. PLoS ONE 2016 Jan 29;11(1):e0147976. [doi: 10.1371/journal.pone.0147976]

4. Kesten S, Maleki-Yazdi R, Sanders BR, Wells JA, McKillop SL, Chapman KR, et al. Respiratory rate during acute asthma. Chest 1990 Jan;97(1):58-62. [doi: 10.1378/chest.97.1.58] [Medline: 2403901]

5. Gobbi A, Dellacá RL, King G, Thamrin C. Toward Predicting Individual Risk in Asthma Using Daily Home Monitoring of Resistance. Am J Respir Crit Care Med 2017 Jan 15;195(2):265-267. [doi: 10.1164/rccm.201603-0537LE] [Medline: 28084825]

6. Bergese SD, Mestek ML, Kelley SD, McIntyre R, Uribe AA, Sethi R, et al. Multicenter Study Validating Accuracy of a Continuous Respiratory Rate Measurement Derived From Pulse Oximetry. Anesthesia \& Analgesia 2017;124(4):1153-1159. [doi: 10.1213/ane.0000000000001852]

7. Charlton PH, Bonnici T, Tarassenko L, Clifton DA, Beale R, Watkinson PJ. An assessment of algorithms to estimate respiratory rate from the electrocardiogram and photoplethysmogram. Physiol Meas 2016 Apr 30;37(4):610-626 [FREE Full text] [doi: 10.1088/0967-3334/37/4/610] [Medline: 27027672]

8. Addison PS. Respiratory effort from the photoplethysmogram. Med Eng Phys 2017 Mar;41:9-18 [FREE Full text] [doi: 10.1016/j.medengphy.2016.12.010] [Medline: 28126420]

9. Bailon R, Sornmo L, LagunaCG-derived RFE. Advanced methods and tools for ECG data analysis. the University of Michigan: Artech House; 2006:E.

10. Meredith DJ, Clifton D, Charlton P, Brooks J, Pugh CW, Tarassenko L. Photoplethysmographic derivation of respiratory rate: a review of relevant physiology. J Med Eng Technol 2012 Jan 20;36(1):1-7. [doi: 10.3109/03091902.2011.638965] [Medline: 22185462]

11. Prinable J, Jones P, Thamrin C, McEwan A. Using a recurrent neural network to derive tidal volume from a photoplethsmograph. 2007 Dec 13 Presented at: IEEE Life Sciences Conference; 2017; Sydney, Australia p. 218-221. [doi: 10.1109/1sc.2017.8268182]

12. Hochreiter S, Schmidhuber J. Long Short-Term Memory. Neural Computation 1997 Nov;9(8):1735-1780. [doi: 10.1162/neco.1997.9.8.1735]

13. Myles P, Cui J. Using the Bland-Altman method to measure agreement with repeated measures. Br J Anaesth 2007 Sep;99(3):309-311 [FREE Full text] [doi: 10.1093/bja/aem214] [Medline: 17702826]

14. Choi A, Shin H. Photoplethysmography sampling frequency: pilot assessment of how low can we go to analyze pulse rate variability with reliability? Physiol Meas 2017 Mar 23;38(3):586-600. [doi: 10.1088/1361-6579/aa5efa] [Medline: 28169836]

15. Charlton PH, Bonnici T, Tarassenko L, Alastruey J, Clifton DA, Beale R, et al. Extraction of respiratory signals from the electrocardiogram and photoplethysmogram: technical and physiological determinants. Physiol Meas 2017 May 03;38(5):669-690. [doi: 10.1088/1361-6579/aa670e] [Medline: 28296645]

16. Addison P, Watson J. Secondary wavelet feature decoupling (SWFD) and its use in detecting patient respiration from the photo- plethysmogram. 2003 Sep 17 Presented at: Engineering in Medicine and Biology Society, 2003. Proceedings of the 25th Annual International Conference of the IEEE. Vol. 3. IEEE, 2003; 2003; Cancun, Mexico. [doi: 10.1109/iembs.2003.1280448]

17. Karlen W, Raman S, Ansermino JM, Dumont GA. Multiparameter Respiratory Rate Estimation From the Photoplethysmogram. IEEE Trans. Biomed. Eng 2013 Jul;60(7):1946-1953. [doi: 10.1109/tbme.2013.2246160]

18. Ruangsuwana R, Velikic G, Bocko M. Methods to extract respiration information from ECG signals. 2010 Jun 28 Presented at: 2010 IEEE International Conference on Acoustics, Speech and Signal Processing; March 14-19, 2010; Dallas, TX. [doi: 10.1109/ICASSP.2010.5495584]

19. Lázaro J, Gil E, Bailón R, Mincholé A, Laguna P. Deriving respiration from photoplethysmographic pulse width. Med Biol Eng Comput 2013 Feb;51(1-2):233-242. [doi: 10.1007/s11517-012-0954-0] [Medline: 22996834]

20. Cui Z, Ke R, Pu Z, Wang Y. Deep Stacked Bidirectional and Uni-directional LSTM Recurrent Neural Network for Network-wide Traffic Speed Prediction. 2016 Presented at: In 6th International Workshop on Urban Computing (UrbComp ); 2017; Chicago, USA.

21. Srivastava N, Hinton G, Krizhevsky A, Sutskever I, Salakhutdinov R. Dropout: a simple way to prevent neural networks from overfitting. The Journal of Machine Learning Research 2014;15(1):1929-1958.

22. Arlot S, Celisse A. A survey of cross-validation procedures for model selection. Statist. Surv 2010;4:40-79. [doi: 10.1214/09-ss054]

23. Prinable JB, Foster JM, McEwan AL, Young PM, Tovey E, Thamrin C. Motivations and Key Features for a Wearable Device for Continuous Monitoring of Breathing: A Web-Based Survey. JMIR Biomed Eng 2017 Jun 26;2(1):e1. [doi: 10.2196/biomedeng.7143]

24. Choi C, Ko B, Lee J, Yoon S, Kwon U, Kim S, et al. PPG pulse direction determination algorithm for PPG waveform inversion by wrist rotation. 2017 Sep 14 Presented at: In Engineering in Medicine and Biology Society (EMBC), 2017 39th Annual International Conference of the IEEE; 2017; Seogwipo, South Korea p. 4090-4093. [doi: 10.1109/embc.2017.8037755]

25. Wolling F, Van Laerhoven K. Fewer Samples for a Longer Life Span: Towards Long-Term Wearable PPG Analysis. 2018 Sep 20 Presented at: In Proceedings of the 5th international Workshop on Sensor-based Activity RecognitionInteraction (p. 5). ACM; 2018; Chicago, USA. [doi: 10.1145/3266157.3266209] 
26. Chang A, Culurciello E. Hardware accelerators for recurrent neural networks on FPGA. 2017 Presented at: IEEE International symposium on circuits and systems (ISCAS); 2017; Baltimore, USA p. 1-4. [doi: 10.1109/iscas.2017.8050816]
Abbreviations
BPM: breaths per minute.
BR: breathing rate.
I:E: inspiration:expiration ratio.
IBI: interbreath interval.
GPU: graphics processing unit.
LoA: limits of agreement
LSTM: long short-term memory.
PPG: photoplethysmogram.
RTV: relative tidal volume.
Texp: expiration period.
Tinsp: inspiration time.
TVW: tidal volume waveform.

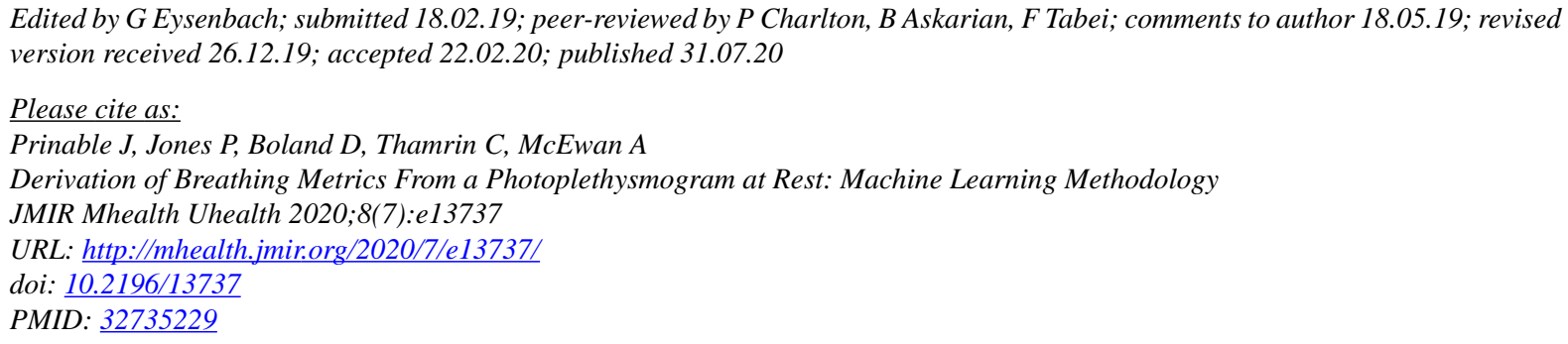

(CJoseph Prinable, Peter Jones, David Boland, Cindy Thamrin, Alistair McEwan. Originally published in JMIR mHealth and uHealth (http://mhealth.jmir.org), 31.07.2020. This is an open-access article distributed under the terms of the Creative Commons Attribution License (https://creativecommons.org/licenses/by/4.0/), which permits unrestricted use, distribution, and reproduction in any medium, provided the original work, first published in JMIR mHealth and uHealth, is properly cited. The complete bibliographic information, a link to the original publication on http://mhealth.jmir.org/, as well as this copyright and license information must be included. 